\title{
THE ATOMIC ENERGY RESEARCH ESTABLISHMENT, HARWELL
}

$T$ HE Atomic Energy Research Establishment was founded by a decision of the Government in November, 1945, and Prof. J. D. (now Sir John) Cockcroft was appointed director in January, 1946. In order to provide a nucleus of accommodation and a prepared site, the permanent R.A.F. aərodrome of Harwell was allocated to the Establishment and building work began there in April, 1946. Harwell was chosen because of its convenient geographical location, combining reasonable access to London with nearness to a large university (Oxford), which was considered to be important so that staff should have good opportunities for discussion with university colleagues.

A nucleus of experienced staff was available in 1946, some of whom had worked in the United States Atomic Energy Project at Los Alamos and Berkeley, while others. had helped to build the Atomic Energy Plant at Chalk River in Canada. These were joined by exp-3rimental electronics staff working at the Telecommunications Research Establishment in Malvern, and by a fow senior members of universities. This nucleus has been added to by the recruitment of junior staff, and there are at present Divisions of Theoretical Physics, Nuclear Physics, General Physjcs, Chemistry, Chemical Engineering, Metallurgy, and Health, each of the size of a large university research school.

The Establishmont has very close links with the universities and industry. Many senior members of the project after the War returned to take charge of university departments; these scientific workers are now consultants to Harwell and some of them serve on the Technical Committee which advises the Department of Atomic Energy. In addition, many of the technological problems of the Establishment are solved by collaboration with industry and with laboratories of Government departments and research associations.

The purpose of the Establishment is to carry out fundamental research and development in atomic energy. It is responsible also for providing scientific and technical information to the Controller of Production, Atomic Energy (Lord Portal) and his engineering organisation at Risley, which is preparing the design for the plutonium production plant which is being built near Sellafield in Cumberland.

\section{Pile Programme}

The first requirement for an atomic energy programme of this nature was to build piles, and the first pile, GLEEP (graphite low energy experimental pile), was completed in August, 1947. It is a slow nuutron graphite moderated reactor developing $100 \mathrm{~kW}$. of nuclear energy. The simplest possible design was chosen so that early experience could be gained in pile operation, and, in fact, the time taken from beginning constructional work to the start-up of the pile was only just over a year.

It has a concrete shield, five feet thick, and the graphite blocks and natural uranium rods are built up within the shield roughly in the form of a cylinder lying on its side. The power-level at which the pile is operating is measured by means of boron trifluoride chambers sunk into the pile, and the current in the chambers suitably amplified is displayed in the control room on a dial calibrated directly in kilowatts. This information is also used to maintain the power of the pile at a constant level by controlling the position of neutron-absorbing cadmium 'control rods', hanging vertically in the pile. A second set of 'shut-off rods' is available to shut down the pile.

GLEEP is being used for measurement of the properties of atomic nuclei, for testing the nuclear properties of materials used in the construction of piles and for the production of radioactive isotopes.

The second pile, BEPO (British experimental pile), started up at 3 p.m. on July 3, 1948. Although not much larger physically than GLEEP, it is a much more complicated engineering structure and is designed to develop $6,000 \mathrm{~kW}$. of nuclear energy. The heat is removed by an airstream which is drawn through the channels in which the uranium rods lie, and exhausted through a $200-\mathrm{ft}$. chimney stack. This pile will be used for studying the effects of irradiation on the structural and physical properties of materials which will be used in future piles, and the much higher neutron flux available makes possible a correspondingly wider range of experiments than in GLEEP. BEPO will also be the main source of radioactive isotopes for Great Britain, and it is expected that this work will be transferred from GLEEP to BEPO towards the end of this year, thus freeing GLEEP for full-time experimental work.

In preparation for building these two piles, it has been necessary to develop the production of pure graphite both in Canada and in Great Britain, and also to produce pure uranium metal and fabricate from it the aluminium-encased uranium rods which are used in the piles. This work has involved a large development programme, all of which has been undertaken outside the Establishment, both by industry and other Government establishments.

After the operation of piles, the next stage in a pile programme is the extraction of plutonium from the irradiated uranium metal, and information on the extraction process will be required for the Sellafield plant. The chemical problems involved in the separation of plutonium from the unused uranium and the highly radioactive fission products are among the most difficult in the atomic energy programme, and for this and other radiochemical work a new laboratory is being built at Harwell. This new radiochemical laboratory is a large two-story building, consisting of a central administrative block, connected by air locks and changing rooms to two wings. The wings contain a number of self-contained laboratory suites, each fitted with lead-lined chambers where all radioactive work will be done. A very large air flow is required through the chambers, equivalent at maximum rate to 120 air changes per minute in the laboratories, and this has led to the provision of an exceptionally large ventilation system, the air ducts for which, together with other services, occupy the whole upper floor of the building. Until this building is complete, temporary laboratories for the work have been provided in one of the original buildings.

Buildings are also being erected for chemical engineers, who will follow up the work of the research chemists with semi-scale plant. The chemical engineering programme includes the study of methods of extraction of uranium from the many kinds of low-grade ores in which it occurs. 


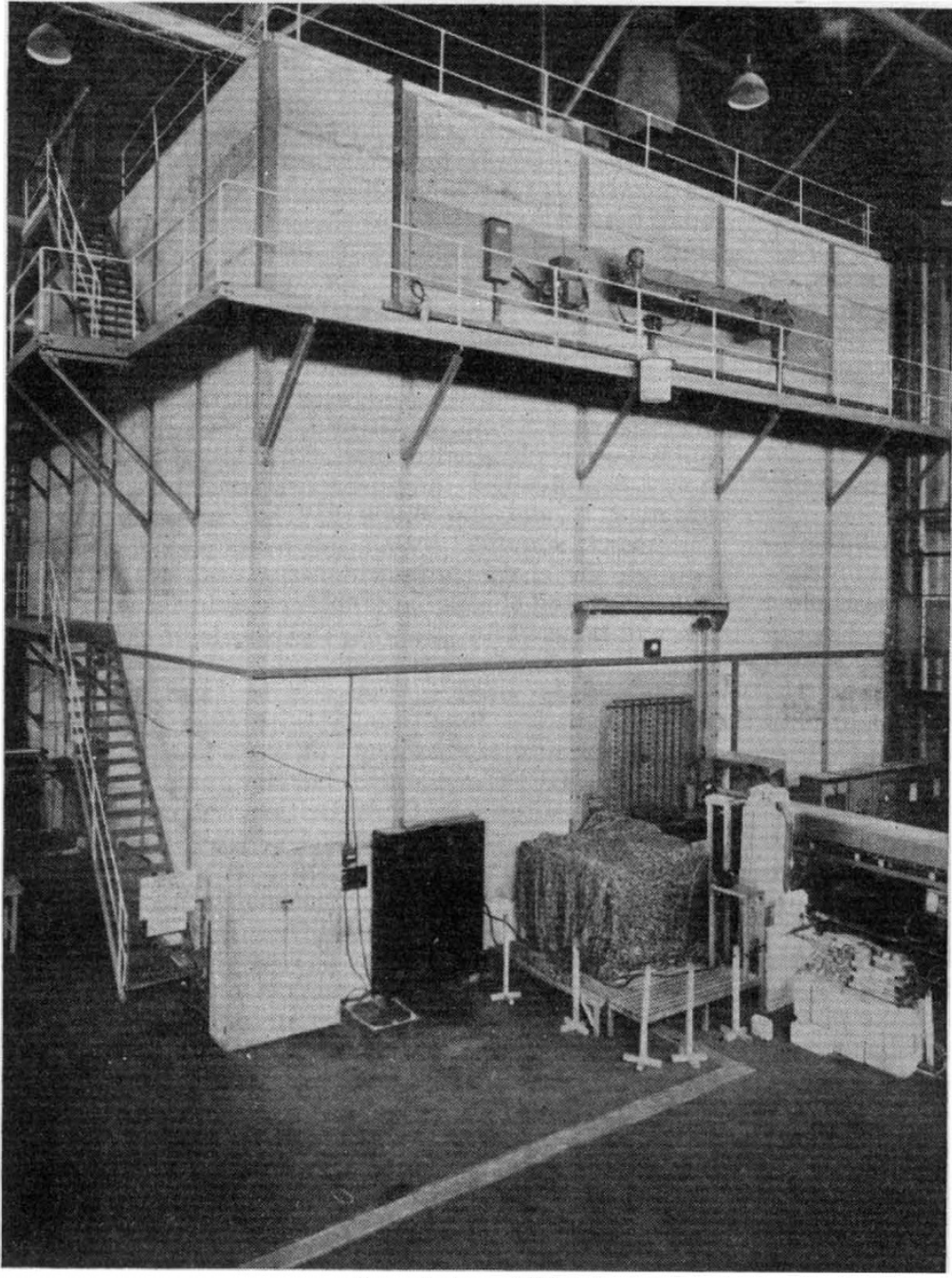

General view of GLEEP

\section{Industrial Applications of Nuclear Power}

The Atomic Energy Research Establishment is working on the problem of the application of nuclear power to industrial purposes. A very small beginning will be to extract from the cooling air stream some of the heat developed by BEPO and to use it for space heating. A heat exchanger for this purpose is being installed.

The design of natural uranium piles in which power could be generated as a by-product at a moderate efficiency is being investigated in detail. This investigation is not complete; but it would appear that the construction of such piles is likely to be technically feasible. They would not, however, be worth while economically since such piles only burn up a very small proportion of the uranium. They would only be worth while as a first step to gain experience.

If nuclear power is to become important in the world, four major problems have to be solved. First, the so-called breeding problem must be solveduranium 235, the only naturally occurring, or 'primary', nuclear fuel must be made to breed 'secondary' fuel from more abundant materials : either plutonium from uranium 238 or U233 from thorium. Second, materials must be found which will stand high temperatures and be suitable for use in pile construction. Third, the chemical extraction problem must be solved-the fissile material produced must be separated economically from the unused uranium or thorium and the intensely radioactive fission products. Fourth, a method of disposal must be found for the large amount of unwanted radioactive material which would be produced by power stations operating on nuclear fuel.

These problems are difficult, and their solution is going to take time. For this reason, no serious person working in the field believes that nuclear energy will make any contribution to world power during the next decade. At present, work is entirely in the theoretical and laboratory stages; but it is probable that a number of pilot plants will be built to allow all these problems to be investig. ated. Until this is done no accurate assessment of the possibilities for the future can be made.

\section{Nuclear Physics}

For fundamental research in nuclear physics the Establishment is being equipped, in addition to the two piles, with several particle-accelerating machines.

A frequency-modulated cyclotron with Il0-in. diameter pole pieces is under construction which will produce particles with a maximum energy of about $200 \mathrm{MeV}$. : it will be completed about the end of this year. This machine will enable the nuclei of most atoms to be broken up, and will be used to produce new types of nuclei which cannot be produced in the piles.

For the production of monokinetic beams of lowerenergy particles, a 5-million volt Van de Graaff generator has been built. The design is similar to that of a machine recently completed by Prof. Van de Graaff at the Massachusetts Institute of Tech. nology, and close contact has been maintained between the Atomic Energy Research Establishment and the Massachusetts Institute of Technology teams. The design voltage has been obtained without the accelerating tube in position, and tests are in progress with accelerating tukes. A 2-MV. machine for work in radiation chemistry has been built and is being tested by the manufacturer.

In addition to machines for work at Harwell, the Establishment is assisting universities and the Medical Research Council by carrying out, on behalf of the Department of Scientific and Industrial Research, development of high-energy particle accelerators for use in universities and medical departments. A group working at the Telecommunications Research Establishment in Malvern is directing the 
development of synchrotrons, including two $30-\mathrm{MeV}$. machines for the Medical Research Council, a $140-\mathrm{MeV}$. machine for the University of Oxford and a $300-\mathrm{MeV}$. machine for the University of Glasgow. It is also developing the design of linear accelerators and recently com. pleted a 4-MeV. machine which is now in great demand for experimental work in nuclear physics and radiation chemistry.

Apart from accelerating machines, a beta spectrometer has been completed with provision for automatic recording, and small groups are working on the design of neutron spectrometers and other problems.

\section{The Isotope Programme}

It has already been announced that GLEEP has been used for the production of radioactive isotopes since September 1947. The rate of production of samples has increased steadily and is now about 150 per month. Altogether more than eight hundred deliveries have been made, mostly to English universities and hospitals and to workers at the Establishment, but including a few to European countries, and one air delivery to South Africa.

It is expected that BEPO will take over the production of radio-isotopes towards the end of 1948. It will then be the main source of radio-isotopes for Great Britain, and will make it possible to extend the supply to countries in the British

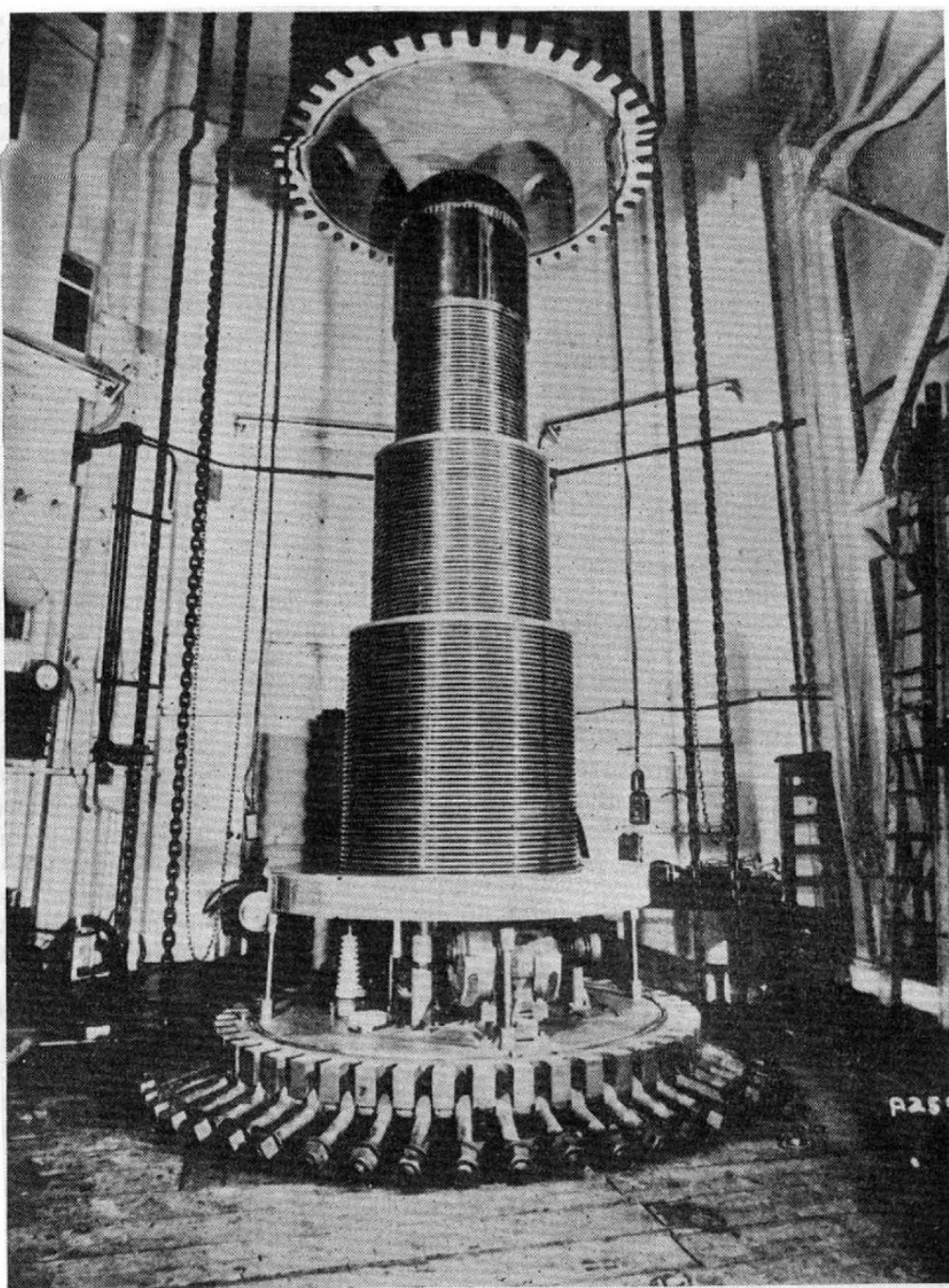

Van de Graaff high-voltage generator
Commonwealth and Europe. The higher neutron flux available will allow the preparation of a much wider range of materials, and of higher activity samples.

It is intended that the chemical processing and delivery of samples, except those with very short half-lives, will be undertaken by the Radio-Chemical Centre at Amersham, and arrangements have been made for the Chemical Research Laboratory to prepare complicated organic molecules containing radio-carbon, when these are required.

In addition to radio-isotopes, the Establishment will produce separated stable isotopes of many elements. Research groups are working on the separation of stable isotopes by several methods, including thermal diffusion, fractional distillation and chemical exchange. A thermal diffusion plant for the preparation of enriched oxygen 18 is being built, which should give by autumn 1948 a yield of $1 / 7 \mathrm{gm}$. of oxygen 18 per week, at a concentration of 20 per cent. A 34-ft. fractional distillation column, working with liquid carbon monoxide, will be complete in a few months time, and should give a yield of $0 \cdot 3 \mathrm{gm} . /$ day of carbon 13 by the end of the year. A smallscale electro-magnetic separator has been built for other elements, and a much larger instrument, capable of separating any elements, is being erected. Several mass spectrometers are available for assaying samples, and some development work on the design of these instruments is being undertaken.

\section{Electronics}

An atomic energy project requires a great variety of electronic instruments, for example, for pile control, radiochemistry, experiments in nuclear physics and health monitoring. A considerable section of the staff is engaged in designing and developing these instruments. In general, the design is carried to a stage where it can be taken over for production by industry, and the Establishment takes the initiative in finding the requirements of university and medical users, so that their needs also can be met in both the design and production stages.

\section{Health}

In view of the possible hazards from radiation and radioactive dusts, very stringent precautions are taken at the Establishment, and the health of all workers is the responsibility of a Medical Division. Everybody is given a thorough medical examination on joining the Establishment, and this is repeated 
periodically. Workers exposed to radiation have their blood examined from time to time to see whether any change occurs in the number of cells, due to exposure to radiation. Safety measures taken have been such, however, that no over-exposure has occurred and there has been no single case of any harmful effects due to radiation.

Arrangements for the monitoring of scientific workers and their laboratories for radioactivity are the responsibility of a Health Physics Group. Staff exposed to radiation carry a photographic film to record the total dosage taken each week, and those working in very active areas carry pocket condenser chambers from which they can see at any time the amount of radiation to which they have been exposed since the chamber was charged. In addition, fixed instruments are placed in the pile buildings, active chemistry laboratories and grounds to provide a continuous record of radiation. Portable instruments are used to make measurements during any operation such as withdrawing materials from a pile. As a result of all these precautions the actual exposure of staff to radiation is well below the levels which have been recommended by the Medical Research Council. The Health Physics Group is also responsible for monitoring the effluent water from the Establishment and seeing that it complies with the rigid specifications laid down by the Medical Research Council.

SOME NEW

RHEOLOGICAL PHENOMENA AND THEIR SIGNIFICANCE FOR THE CONSTITUTION OF MATERIALS

\author{
By S. M. FREEMAN \\ AND \\ K. WEISSENBERG* \\ British Cotton Industry Research Association
}

$\mathrm{T}$ HE materials studied in rheology are of varied constitution, with intricate patterns of constituent components of visible, colloidal, molecular, atomic and sub-atomic sizes. The patterns range from a coarse-grained type in which a discontinuous structure is apparent, to a fine-grained one which appears homogeneous and continuous to the eye and even to the microscope; the former type is represented by biological tissues, textile materials, natural and synthetic sponges, foams, powders, timber, cement, etc., and the latter by gases, amorphous liquids and solids, single crystals, etc. In the engineering constructions of Nature and man, widespread use is made of coarse- and fine-grained materials, and this invites an investigation into their mechanical properties as exhibited in changes of volume and form, and into the relation between these properties and the constitution of the materials.

In the present article we shall discuss experiments in which we use both types of material, the coarse-grained ones being particularly valuable here because they can be made to give combinations of mechanical properties suited to the purpose in hand and not easily available in the fine-grained types.

* Present address: British Rayon Research Association, Bridgewater House, Whitworth Street, Manchester 1.
Moreover, one can easily vary the constitution of a coarse-grained material by physical means and observe macroscopically how the mechanical properties depend on the constitution. As an example, consider coarse-grained sponges of rubber or rubberlike substances. The sponges can be made to display large elastic recoveries of strains for changes of both volume and form, a combination not found in finegrained materials. Moreover, the sponges can be made in various structural designs, including the extremes of an open structure with intercommunicating holes, and a closed one with isolated holes, and also covering a wide range of mixed structures in which the holes may be filled with air or viscous or visco-elastic liquids. Another example is provided by the coarse-grained assemblies of fibres, ranging from single hairs to textile fabrics, and yet another by powders, etc. We selett from the materials of all types samples of a size sufficiently large to allow a determination of the mechanical properties in terms of a continuum theory, the coarse- and finegrained structures alike being averaged out into continua over regions of macroscopic, microscopic or submicroscopic dimensions. For the coarsegrained materials we can then observe simultan. eously the mechanical properties as defined by the continuum theory, and the constitution as revealed by the structural pattern of the components of macroscopic size, and thus have a means of correlating one with the other. For the fine-grained materials we can observe the mechanical properties as before, and elucidate by analogy the correlation with the hidden constitution on a microscopic and submicroscopic scale.

For ease of reference we shall introduce a nomen. clature suited to distinguish between different kinds of response to mechanical actions. We have examined all the materials first in their natural state, when they are at rest and free from externally applied forces, and we use the term 'isotropic' to indicate that the material in its natural state displays full spherical symmetry in response to actions along the various directions in space ; the term 'compressible' is used to indicate appreciable changes in volume within the range of actions considered; and the term 'shear elastic' to denote in that range an appreciable amount of shear strain recovery under a sudden release of all external forces. By contrast, we use the term 'anisotropic' for a deviation from spherical symmetry, and the terms 'incompressible' and 'shear inelastic' when the changes of volume and of shear strain recovery respectively are inappreciably small.

\section{Continuum Theory of the Elasticity of Volume and Form}

In the discussion of a continuum theory of the elasticity of volume and form, we shall restrict ourselves to materials which are isotropic in their natural state, since the theory appears for these materials in its simplest form.

We consider here a differentially small portion of an elastic material. Under an applied load of external forces, the portion will pass from its natural state to some static state of equilibrium, shortly referred to as the 'loaded state', and will appear there as a cube with edges of unit length. In transit from the natural state the cube will have acquired in the loaded state a certain strain, according to the changes in volume and form, and a certain stress, according to the 